\title{
Congenital Aortic Stenosis in a German Shepherd - Case Report
}

\author{
Iuliu SCURTU ${ }^{1 *}$, Gavril GIURGIU ${ }^{1}$, Cristian POPOVICI ${ }^{1}$, Florin BETEG ${ }^{2}$, Mircea MIRCEAN ${ }^{1}$ \\ ${ }^{1}$ Faculty of Veterinary Medicine, Internal Medicine Department, 3-5 Manastur street, Cluj-Napoca, \\ Romania \\ ${ }^{2}$ Faculty of Veterinary Medicine, Department of Surgery, 3-5 Manastur street, Cluj-Napoca, Romania \\ *Corresponding author, e-mail: icscurtu@yahoo.com
}

Bulletin UASVM Veterinary Medicine 71(2) / 2014,

Print ISSN 1843-5270; Electronic ISSN 1843-5378

DOI:10.15835/buasvmcn-vm: 10512

\begin{abstract}
Aortic stenosis is a congenital heart disorder that occurs mainly in large breed dogs. Many large breed dogs were diagnosed with this congenital heart disorder, but Golden Retriever, Rottweiler, Boxer, German shepherd and Newfoundland seem to be overrepresented. Aortic stenosis may manifest itself as supravalvular, valvular and subvalvular. The subvalvular form (subaortic stenosis) is the most common form in the dog. With this defect, a fibrous band or ring located just below the aortic semilunar valves impedes left ventricular emptying.

Aims: We want to underline clinical and paraclinical abnormalities associated with this disease.

Four month old pure German shepherd female was brought for specific cardiac evaluation at our unit. Clinical investigation was performed after a specific algorithm (evaluation of precordial thrill, pulse and murmur, assessment of lung problems). Paraclinical investigations included electrocardiography and echocardiography.

Clinically point of view we identified a precordial thrill on the left thorax. On auscultation we identified a systolic murmur located cranially, at the base of the heart, area which corresponds with aortic orifice location. The murmur's grade was 6/6. Electrocardiographically we observed high amplitude of R waves with supraventricular and ventricular (bigeminy) rhythm abnormalities. On echography we underlined an increase of myocardium echogenity with hyperechoic foci, localized mainly in left ventricular free wall, decreased left ventricular chamber with thickness of interventricular septum and left ventricular free wall. Doppler echography identified a turbulent jet through aortic orifice with $5.5 \mathrm{~m} / \mathrm{s}$ velocity. Based on clinical and paraclinical tests diagnostic is severe aortic stenosis.

Congenital aortic stenosis implies a severe diagnostic. Doppler investigation is the most important tool in aortic stenosis diagnostic.
\end{abstract}

Key words: aortic stenosis, congenital heart disease, Doppler, echocardiography

\section{INTRODUCTION}

Aortic stenosisis the second mostfrequent type of congenital heart disorder in dogs. Aortic stenosis has three categories of abnormalities, depending on localization of stenosis (supravalvular, valvular and subvalvular) (Linde, 2006). Evaluating pressure gradient across the aortic valve, aortic stenosis is categorized as mild, moderate and severe. The subvalvular form (subaortic stenosis) is the most common form in dogs. In this instance, a fibrous band localized under the aortic ring impedes left ventricular outflow (Tilley, 2008). Valvular aortic stenosis is rarely seen as a single entity, but occurs in conjunction with subvalvular obstruction. Supravalvular stenosis is also very rarely diagnosed, mainly in cats (Boon, 2011). The most frequent affected breeds are: Newfoundland (a polygenic inheritance pattern has been identified), Boxer, English Buldog, German Shepherd, Golden Retriever, Great Dane, Rottweiller, and Samoyed (Tilley, 2008). Subaortic stenosis in Newfoundland dogs has been graded in postmortem studies as follows: grade 1 - small, whitish, slightly raised nodules on the endocardial surface of the ventricular septum, immediately below the aortic valve; grade 2 - narrow ridge 
of whitish, thickened endocardium extending partially about the left ventricular outflow tract; grade 3 - the most severe form, a fibrous band, ridge, or collar completely encircling the left ventricular outflow tract, just below the aortic valve (Fox et al., 1999).

\section{MATERIALS AND MEHODS}

\section{Case Presentation - History}

Four months old, female, pure German shepherd breed was brought for cardiologic examination at our unit. Clinical signs had been expressed for one month before presentation to our department. Dyspnea, fatigue, effort intolerance, collapsing episodes and unwillingness to play were the chief complains. The dog also presented coughing and vomiting. The owner related that the parents of the dog were rigorously selected to underline some body features. Two day before presentation to our department, the patient had refused to eat. The owner also declared that the dog is smaller than her littermates.

\section{Clinical signs}

Clinical examination identified a patient with severe dyspnea, labored breathing, having a good body condition. Mucous membranes were greys and the dog had a weak pulse, being difficult to estimate frequency. Lung auscultation revealed crackles over both hemithorax. Palpation of heart projection area confirmed the presence of the precordial trill, and we also identified a systolic murmur with PMI (point of maximum intensity) over left cranial base. Murmur was scaled as $6 / 6$ and also spread to right thorax.

\section{ECG}

We identified high amplitude of $\mathrm{P}$ waves $(0.6 \mathrm{mV})$ and $\mathrm{R}$ waves $(4.2 \mathrm{mV})$ in DII, with supraventricular and ventricular abnormalities. Ventricular abnormality had bigeminy pattern with an aspect of right bundle branch block, aspect consistent with left ventricular origin of the stimulus (fig. 1). MEA (mean electric axis) was within reference range intervals.

\section{Ecocardiography}

On ultrasound examination we identified an increase of myocardium echogenicity, with hyperechoic foci localized mainly within left ventricular wall with enlargement of papillary muscles. Left ventricular wall and interventricular septum, as well, were thicker than normal. Left atrium:aorta (LA:Ao) ratio was higher than normal
(1.7). Aortic cusps seemed less mobile, and a structure located below aortic valve prolapsed within left ventricular outflow tract. Color study imaged a mitral regurgitation jet and non-laminar flow within aorta (left apical four and five chambers view). Continuous flow Doppler interrogation or aortic valve imaged a high velocity jet, about 5.5$6.0 \mathrm{~m} / \mathrm{s}$ (left apical five chambers view).

All the aspects found during clinical and paraclinical examinations were consistent with severe aortic stenosis.

\section{RESULTS AND DISCUSSIONS}

Aortic stenosis occurs mainly in large breed dogs and is the second most frequent congenital heart disease in dogs. There are three forms of aortic stenosis; subvalvular stenosis being the most frequently diagnosed in dogs. In contrast to dogs, cats rarely develop this type of abnormality. Clinical examination could be predicted for this type of pathology. There are clinical features that could be suggestive for aortic stenosis, but a definitive diagnostic implies other exams. Systolic murmur with PMI over left cranial base associated with a weak pulse could be associated with this type of pathology, but these signs are not pathognomonic with aortic stenosis. In Boxer, Linde (2006) also suggests that identification of high grade murmur on auscultation represents an inexpensive tool for diagnosis orientation in case of aortic stenosis. Low grade murmur may, however, be an insignificant finding, and would require an echocardiographic exam to either confirm or rule out the presence of abnormal aortic velocity. In one study performed by Schober (2002), $42 \%$ of dogs with AS had a grade of $2 / 6$ systolic ejection-type heart murmur, $33 \%$ had a grade of $3 / 6,12 \%$ had a grade of $4 / 6,12 \%$ had a grade of $5 / 6$, and $1 \%$ had a grade of $6 / 6$ systolic murmur heard best over the left hemithorax. Main clinical complaints were: exercise intolerance in $23 \%$, syncope in $16 \%$, chronic loss of body weight in $4 \%$ and exercitional cyanosis in $3 \%$ of dogs (Schober, 2002).

ECG identifies supraventricular and also ventricular rhythm disturbances. Premature supraventricular contractions could be related with atrial dilation; ventricular premature contractions occur mainly in bigeminy pattern. The origin of focus that triggers ectopic beats seems to be in left ventricle, ectopic beats being negative in DII. 


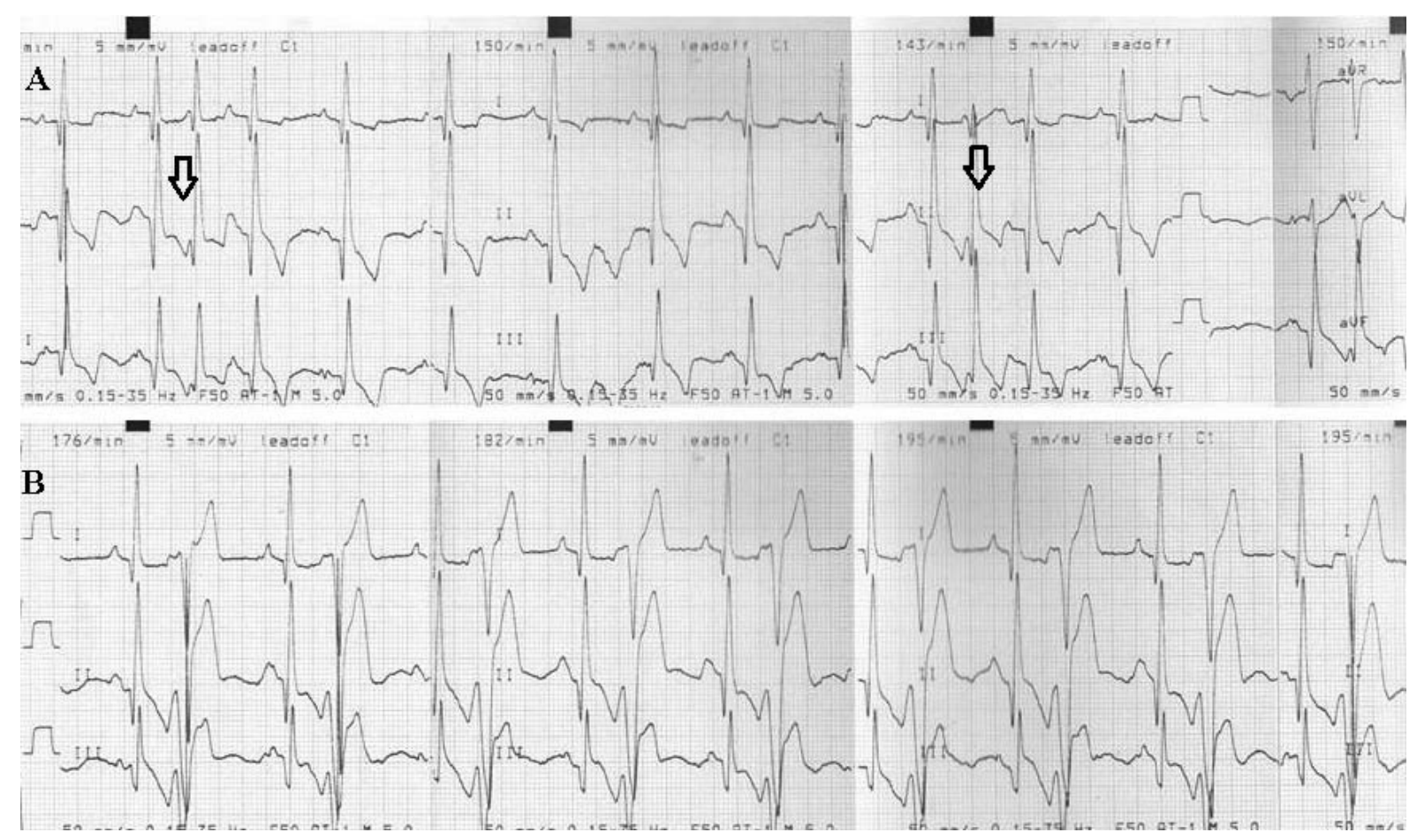

Fig. 1. Supraventricular ectopic beats (strip A). Ventricular bigeminy (strip B)

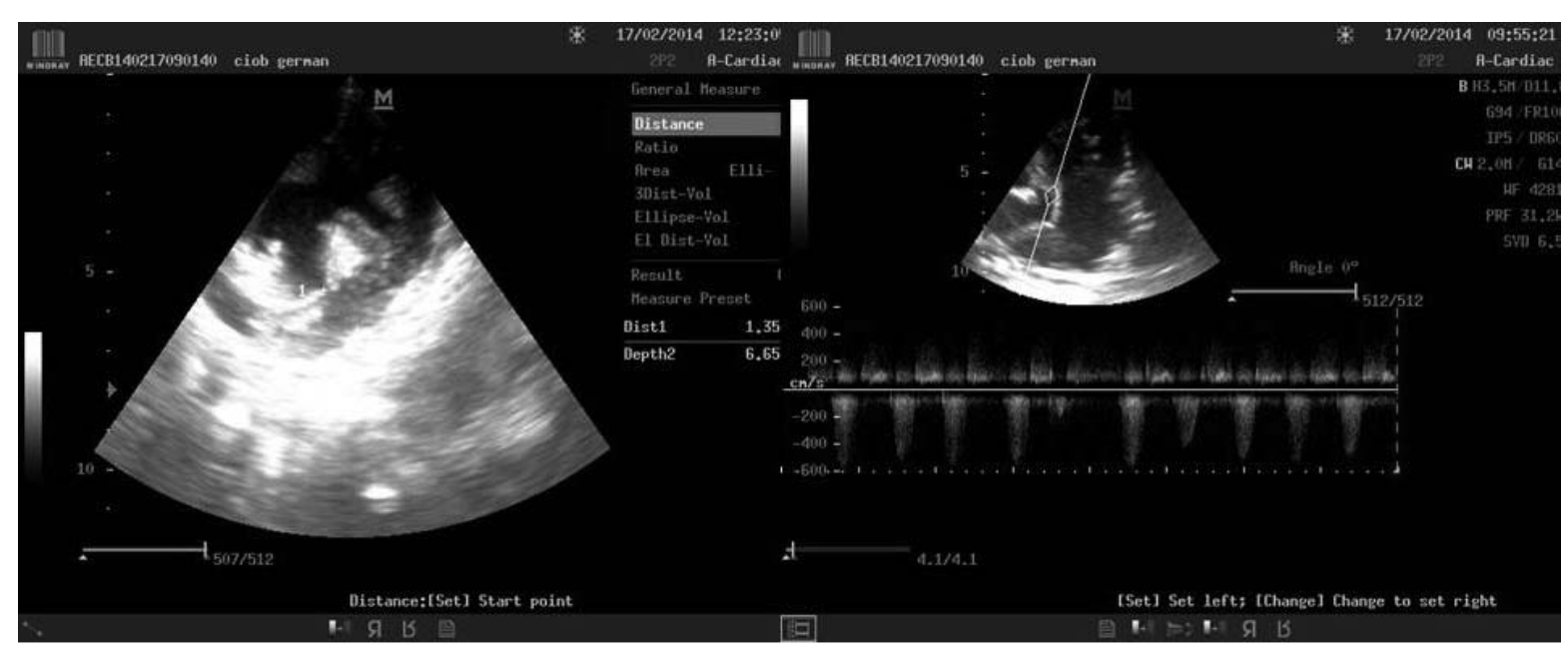

Fig. 2. Increased echogenicity of LV wall, enlarged papillary muscle. Peak of aortic flow velocity of $5.5 \mathrm{~m} / \mathrm{s}$.

We also identified an increase of $\mathrm{P}$ wave duration aspect that is consistent with left atrial dilation, and a very tall $R$ wave aspect which is related with left ventricle concentric/eccentric hypertrophy. Linde (2006) sustains that ECG abnormalities (ventricular premature contraction - VPC), in case of AS (aortic stenosis), occur only in severe cases. Mild to moderate AS cases usually are not associated with any rhythm disturbance or with left ventricular ECG pattern (Linde, 2006).

The most consistent findings in case of AS are obtained from echography (Hoglund, 2007). In our case, 2 D echography underlined left ventricular concentric hypertrophy with hyperechoic foci, mainly within left ventricular free wall. Pupillary muscles were enlarged with hyperechoic foci, 
aspect that could suggest muscle mineralization (fig. 2).

LA:Ao ratio was also increased, due to enlargement of left atrium. We also identified an impairment of diastolic function. The majority of dogs with moderate or severe AS had echocardiographic evidence of disturbed LV (left ventricle) diastolic performance, whereas most dogs with mild AS had not. Left ventricular hypertrophy has been shown as the main pathophysiologic substrate for altered LV diastolic function in people with AS (Schober, 2002). The thickeness of the interventricular septum (IVS), as well as the diameter of the left ventricular free wall (LVPW), were significantly related to the severity of AS (Schober, 2002).

We identified a turbulent jet through aortic valve; the alised color signal helps identify the length and location of a subvalvular obstruction (Boon, 2011). We recorded a peak velocity within aorta of $5.5 \mathrm{~m} / \mathrm{s}$ (fig. 2). High velocity flow obtained through the left ventricular outflow tract or aorta is the defining feature of obstruction to outflow (Boon, 2011). The flow profile is somewhat different from that seen with normal aortic flow. Peak velocity is reached closer to the midpoint of ventricular systole as opposed to within the first third of systole. The flow profile becomes more symmetrical as the pressure gradient increases
(Boon, 2011). Aortic stenosis is considered mild when pressure gradient is less than $50 \mathrm{~mm} \mathrm{Hg}$, moderate when pressure gradient is $50-80 \mathrm{~mm}$ $\mathrm{Hg}$ and severe when pressure gradient exceeds $80 \mathrm{mmHg}$. Subvalvular stenosis is a progressive disease and severity may increase until the animal is fully grown (Boon, 2011).

\section{CONCLUSIONS}

Congenital aortic stenosis implies a severe diagnostic. Doppler investigation is the most important tool in aortic stenosis diagnostic.

\section{REFERENCES}

1. Boon J (2011). Veterinary echocardiography. $2^{\text {nd }}$ edition Wiley-Blackwell 490-494.

2. Fox P, Sisson D, Moise S (1999). Textbook of canine and feline cardiology: principles and clinical practice $2^{\text {nd }}$ edition Saunders.

3. Hoglund K, Bussadori C, Domenech O, Haggstrom J (2007). Contrast echocardiography in Boxer dogs with or without aortic stenosis. J of Vet Card 9:15-24.

4. Linde A, Koch J (2006). Screening for aortic stenosis in the Boxer: Auscultatory, ECG, blood pressure and Doppler echocardiographic findings. J of Vet Card 8:79-86.

5. Schober K, Fuentes V (2002). Doppler echocardiographic assessment of left ventricular diastolic function in 74 boxer dogs with aortic stenosis. J of Vet Card 4(1):7-16.

6. Tilley L, Smith Jr. F, Oyama M, Sleeper M (2008). Manual of canine and feline cardiology $4^{\text {th }}$ edition Sauders Elsevier, 223-227. 